\title{
Place Identifier
}

National Cancer Institute

\section{Source}

National Cancer Institute. Place Identifier. NCI Thesaurus. Code C93993.

A unique symbol that establishes identity of the place. 